\title{
Easy, Fast, and Energy-Efficient Object Detection on Heterogeneous On-Chip Architectures
}

\author{
EHSAN TOTONI, MERT DIKMEN, and MARÍA JESÚS GARZARÁN, University of Illinois \\ at Urbana-Champaign
}

\begin{abstract}
We optimize a visual object detection application (that uses Vision Video Library kernels) and show that OpenCL is a unified programming paradigm that can provide high performance when running on the Ivy Bridge heterogeneous on-chip architecture. We evaluate different mapping techniques and show that running each kernel where it fits the best and using software pipelining can provide 1.91 times higher performance and $42 \%$ better energy efficiency. We also show how to trade accuracy for energy at runtime. Overall, our application can perform accurate object detection at 40 frames per second (fps) in an energy-efficient manner.
\end{abstract}

Categories and Subject Descriptors: D.1.3 Concurrent Programming-Parallel Programming; I.4.8 Scene Analysis-Object recognition; C.1.3 Other Architecture Styles-Heterogeneous (hybrid) systems

General Terms: Design, Languages, Performance

Additional Key Words and Phrases: OpenCL, SIMD, heterogeneous on-chip architectures, Energy efficiency, portable (mobile) devices

\section{ACM Reference Format:}

Totoni, E., Dikmen, M., and Garzarán, M. J. 2013. Easy, fast, and energy-efficient object detection on heterogeneous on-chip architectures. ACM Trans. Architec. Code Optim. 10, 4, Article 45 (December 2013 ), 25 pages.

DOI: http://dx.doi.org/10.1145/2555289.2555302

\section{INTRODUCTION}

Many computing platforms used by consumers are portable devices such as notebooks, tablets, smartphones, and more. Since these devices are usually battery powered, achieving high energy efficiency is a crucial challenge. On the other hand, because of their portability, mobile devices encounter many situations where they are expected to understand their environment in a natural way. For example, many photo applications need to automatically adjust the focal range based on the size of faces looking at a camera. In addition, gestures are frequently preferred to classical keyboard- and mouse-based input. Furthermore, search engines can allow a query to be formulated using visual inputs without requiring the user to provide the semantic translation of the visual content. Most natural interactions, such as the examples mentioned, require some usage of vision and video analytics algorithms. These tend to be floating-point

New Article, Not an Extension of a Conference Paper.

This work has been done at the Intel-Illinois Parallelism Center of Department of Computer Science at the University of Illinois at Urbana-Champaign, Urbana, IL, 61801.

Author's addresses: E. Totoni, University of Illinois at Urbana-Champaign - Computer Science; email: totoni2@illinois.edu.

Permission to make digital or hard copies of part or all of this work for personal or classroom use is granted without fee provided that copies are not made or distributed for profit or commercial advantage and that copies show this notice on the first page or initial screen of a display along with the full citation. Copyrights for components of this work owned by others than ACM must be honored. Abstracting with credit is permitted. To copy otherwise, to republish, to post on servers, to redistribute to lists, or to use any component of this work in other works requires prior specific permission and/or a fee. Permissions may be requested from Publications Dept., ACM, Inc., 2 Penn Plaza, Suite 701, New York, NY 10121-0701 USA, fax +1 (212) 869-0481 or permissions@acm.org.

(c) 2013 ACM 1544-3566/2013/12-ART45 $\$ 15.00$

DOI: http://dx.doi.org/10.1145/2555289.2555302 
intensive and computationally demanding but also regular, which make them good candidates for parallelism.

Such data parallel algorithms adapt well to GPU-type architectures, resulting in higher performance and energy efficiency [Kumar et al. 2005]. However, generalpurpose programming of GPUs requires knowledge of new programming paradigms, such as CUDA and OpenCL, which decreases programmer productivity.

Traditionally, the GPU has been a peripheral component, used as a computational aid to the CPU (which is needed for latency-oriented functions such as the operating system). However, deploying stand-alone GPUs may not be desirable (or even practical) for portable platforms for different reasons. First, using an extra chip increases the system design and implementation cost significantly. Second, the extra chip, along with its associated overheads such as power supplies, increases the power and energy consumption. Third, the off-chip connection between the CPU and the GPU may have high performance and energy overheads.

A reasonable alternative for deploying a GPU is to put it on the same chip as the CPU and create a heterogeneous on-chip architecture. Advancements in system-onchip design and increases in the number of available on-chip transistors has made hybrid architectures practical. Emerging examples such as Intel Ivy Bridge [Damaraju et al. 2012], AMD Fusion [Foley et al. 2012], and NVIDIA Tegra 250 [NVIDIA 2011] have implemented this idea.

For this study, we have chosen an application, object detection using ViVid [Dikmen et al. 2012], as a representative of vision applications. The domain of our study is on-chip hybrid architectures, which are most predominantly found in mobile platforms. We believe that object detection is a representative mobile application because it is fairly computationally demanding and it processes streamed visual input from a camera. Similar to our study, most vision applications that would be utilized in mobile devices (e.g., recognition, tracking, stabilization) consist of a pipeline of small numbers of kernels, where kernels are the core compute-intensive components of an application. Of course, there is a large variety of kernels across the spectrum of vision applications. However, from a computational perspective, the pipeline in this article provides a good mixture of kernels efficient on the GPU, CPU, or both. In addition, object detection is an important application for future portable devices, which has not yet been realized beyond basic face detection. Notice that our focus on one application allows us to go deeper into the details of individual kernels. We describe and evaluate the steps one might take to improve performance and energy efficiency: (1) code optimization, (2) mapping strategies, (3) dynamic voltage and frequency scaling (DVFS) [Mudge 2001], and (4) algorithmic tradeoff of accuracy. We report the lessons learned, which would give insight to application developers and system designers.

In this article, we evaluate and analyze different programming paradigms and strategies for energy efficiency. We implement and execute (on the Ivy Bridge architecture) four different code versions of ViVid using (1) OpenCL, (2) OpenMP + auto-vectorization, (3) OpenMP + vector intrinsics, and (4) the OpenCV vision library. The OpenCL version runs on both the CPU and the GPU, while the other versions only run on the CPU. Our experimental results show that OpenCL does not deliver the performance that can be attained when using lower-level interfaces (e.g., vector intrinsics on the CPU) but provides a reasonable performance (Section 4). The OpenCL code processes 40 frames per second (fps) for accurate object detection (Section 5), so it can be used for applications that require real-time object detection (33fps). Notice that the performance of our OpenCL implementation is superior or similar to recent works using much more capable discrete GPUs [Beleznai et al. 2011; Zhang and Nevatia 2008].

We also show that mapping each kernel to the device (CPU or GPU) where it executes more efficiently and overlapping the computation of the kernels is the best approach. Our results show that with these heterogeneous platforms it is possible to 
find mappings that, while executing relatively faster, are less energy efficient (this is discussed in Section 5). In addition, it is possible to gain better energy efficiency by sacrificing a small amount of accuracy algorithmically. For our application, we can reduce $20 \%$ of the energy consumed at the cost of an increase of only $1 \%$ miss-rate on image detection (Section 5.3).

Note that manufacturers do not know how to design hardware and software of future portable devices to support new interfaces (e.g., for human interaction). For instance, specialized hardware accelerators and optimized vision libraries are considered. We show that using a unified programming paradigm (e.g., OpenCL), vision applications can deliver the required performance (for a typical number of frames per seconds) and energy efficiency on heterogeneous on-chip architectures. To the best of our knowledge, the literature only considers large discrete GPUs with very different tradeoffs in performance and energy efficiency for these applications.

The rest of this article is organized as follows: Section 2 describes our application and experimental setup briefly. Section 3 evaluates and analyzes different optimizations for our kernels using OpenCL for the CPU and the GPU. Next, Section 4 compares the performance and programming effort of the OpenCL paradigm to others for the CPU. After that, Section 5 evaluates the performance and energy consumption of different kernels on either the CPU or GPU. It also optimizes the full application's performance and energy consumption using different mapping methods. Finally, Section 6 reviews some related work, and Section 7 concludes the article.

\section{ENVIRONMENTAL SETUP}

\subsection{ViVid}

We focus our study on an object (e.g., face) detection algorithm [Dikmen et al. 2012] for finding objects with a specific shape or appearance in unconstrained visual input. This object detector is analogous to most practical approaches [Jones and Viola 2003; Felzenszwalb et al. 2010] to this problem, which follow a common workflow called "sliding window object detection." This process involves describing the visual information inside small rectangular regions of the image or video frame hypothesized to contain the object and applying a decision function that yields a binary output indicating the presence or absence of the object in each of such rectangles. Sliding window detection is the most established approach for the unconstrained object detection problem. Other popular methods include generalized voting frameworks [Maji and Malik 2009] or contour matching [Ma and Latecki 2011]. In all cases, object detection is a very computationally demanding application because image information needs to be evaluated densely over all the potential locations that may contain the object. ViVid's sliding window approach breaks the problem into two distinct parts: (1) describing the image information and (2) classifying it. The image information is described by correlating the grayscale image with numerous $3 \times 3$ patterns and summarizing these correlations in terms of spatially local histograms. The classification is achieved by processing these histograms through linear support vector machines [Burges 1998].

Other than object detection, there are numerous applications of computer vision on mobile devices including video stabilization, panorama stitching, gesture recognition, and so forth. However, the data description followed by a data association workflow is a common pattern. Typically, the data description part touches every pixel at least once and builds a summarization of structures of interest (e.g., colors, gradients, textures). The data association part measures the distance between the data summaries against stored exemplars. In classification applications, these can be templates for objects, and in segmentation applications these are usually cluster centers. The computational stages in a mobile computer vision application may be computationally balanced, or particular stages may give rise to performance bottlenecks. In our selected object 
detection algorithm, the data description and data association steps are well balanced in terms of their computational load. Therefore, we believe it comprises a good case study with challenges in both stages.

To build our object detector pipeline, we use the ViVid library. ${ }^{1}$ ViVid includes several atomic functions common to many vision algorithms. We have used ViVid successfully in event detection applications [Dikmen et al. 2008; Yang et al. 2009].

For the purposes of this work, we extended ViVid by adding OpenCL equivalents of several kernels. We use the C++ interface to orchestrate the calls to these OpenCL functions or kernels.

\subsection{Blockwise Distance}

This kernel needs to find the maximum response (normalized cross-correlation) of 100 filters on a small square image patch (in this application, $3 \times 3$ ) centered at every pixel of the image while remembering which filter delivered this maximum at every pixel. Algorithm 1 outlines the overall algorithm.

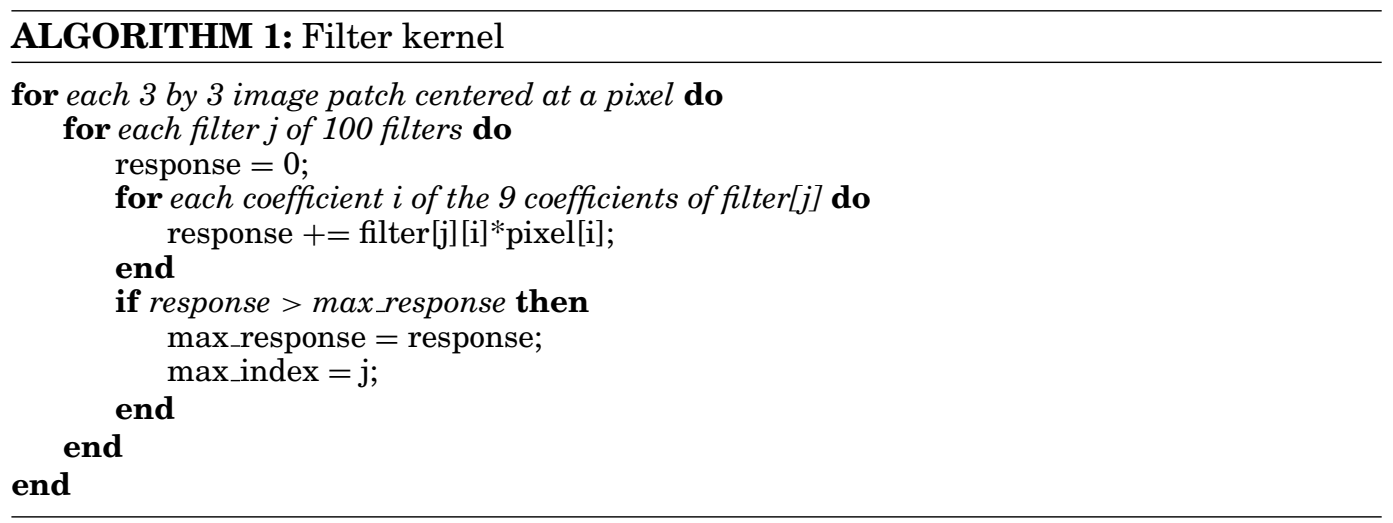

\subsection{Cell Histogram Kernel}

The cell histogram kernel is the second stage of data description, where the lowlevel information collected by the filter kernel is summarized for small, nonoverlapping square blocks of the image. A 100-bin histogram is populated for each of these blocks by accumulating the "max_response" values in their respective bins (given by "max_index") from every pixel inside the block. Note that this operation is different from the well-known image histogramming problem, for which many parallel implementations exist. Our approach differs in two important aspects: (1) the histogram bins represent a weighted sum (not a simple count) and (2) we build many local histograms, not a single global one.

\subsection{Pairwise Distance}

This kernel is the data association step in our application. It finds the Euclidean distance between two sets of vectors, where one vector corresponds to the histogram previously generated and the other vector represents the template. This kernel measures how close each descriptor is to the template of the object of interest. If the distance is small enough, it shall output a detection response.

The kernel is structurally similar to the matrix multiply operation, which finds the dot product between every row of one matrix and every column of another one. However,

\footnotetext{
${ }^{1}$ http://www.github.com/mertdikmen/vivid.
} 
Table I. Intel Ivy Bridge (Core i5 3350 \& 3317U) Processor Specifications

\begin{tabular}{|l|r|r|}
\hline Platform & \multicolumn{1}{|c|}{ Desktop } & \multicolumn{1}{|c|}{ Ultrabook } \\
\hline Processor Number & i5-3550 & i5-3517U \\
\hline \# of Cores & 4 & 2 \\
\hline Base Clock Speed & $3.3 \mathrm{GHz}$ & $1.7 \mathrm{GHz}$ \\
\hline Max Turbo Frequency & $3.7 \mathrm{GHz}$ & $2.6 \mathrm{GHz}$ \\
\hline Base CPU peak & $105.6 \mathrm{GFLOPs}$ & $27.2 \mathrm{GFLOPs}$ \\
\hline Max CPU peak & $118.4 \mathrm{GFLOPs}$ & $41.6 \mathrm{GFLOPs}$ \\
\hline Cache Size & $22 \mathrm{MB}$ & $3 \mathrm{MB}$ \\
\hline Lithography & $77 \mathrm{~W}$ & $22 \mathrm{~nm}$ \\
\hline Max TDP & 2500 & $17 \mathrm{~W}$ \\
\hline Intel HD Graphics & 6 & 4000 \\
\hline GPU Execution Units & $650 \mathrm{MHz}$ & 16 \\
\hline GPU Base Frequency & $1.15 \mathrm{GHz}$ & $1.05 \mathrm{GHz}$ \\
\hline GPU Max Dynamic Frequency & $31.2 \mathrm{GFLOPs}$ & $44.8 \mathrm{GFLOPs}$ \\
\hline Base GPU peak & $55.2 \mathrm{GFLOPs}$ & $134.4 \mathrm{GFLOPs}$ \\
\hline Max GPU peak & &
\end{tabular}

Table II. Software Environment Used for Experiments

\begin{tabular}{|l|c|}
\hline Operating System & Windows 8 Build 9200 \\
\hline GPU driver & Intel 9.17.10.2867 \\
\hline OpenCL SDK & Intel 3.0.0.64050 \\
\hline Compiler & Intel ICC 13.0.1.119 \\
\hline
\end{tabular}

in pairwise distance, we compute the square of the two values' differences, instead of just multiplying them.

\subsection{Ivy Bridge Architecture}

For the experiments reported in this article, we use the two different platforms shown in Table I, both based on the Intel Ivy Bridge architecture. The first one is a $3.3 \mathrm{GHz}$ quadcore used for Desktops and the second one is a $1.7 \mathrm{GHz}$ dual-core used for Ultrabooks. Both platforms have an integrated GPU that can be programmed using OpenCL. ${ }^{2}$ GPUs exploit the Single Instruction Multiple Thread (SIMT) type of parallelism by having an array of Compute Units (CUs). Each CU is assigned a workgroup, where work items in each group run in lock-step, executing the same instruction on different data. GPUs are designed to efficiently exploit data parallelism. Branchy codes may run poorly on GPUs, as all the different paths in a control flow need to be serialized. Note that the Ivy Bridge's GPU is simpler than Nvidia [Lindholm et al. 2008] or AMD/ATI [Zhang et al. 2011] GPUs. It has a small number of compute units and simpler memory hierarchy, for instance.

The Ivy Bridge CPU contains multiple cores, where each core supports advanced vector extensions (AVXs) that apply the same instruction on multiple data simultaneously. AVX supports 256-bit-wide vector units that allow vector operations to operate on eight floating-point numbers simultaneously. Unless otherwise stated, the experiments reported in this article use the Ultrabook platform. For comparison purposes, we have also run experiments on the Desktop platform and an Nvidia Fermi GPU.

For the evaluation, we use Intel SDK for OpenCL [Int 2013a] to run OpenCL codes. We also wrote OpenMP code with and without vector intrinsics that we compiled using the Intel ICC compiler and /O3 compiler flags. Table II summarizes the software environment we use for the experiments. OpenCL is a unified programming paradigm,

$\overline{{ }^{2} \text { http://www.khronos.org/opencl/. }}$ 
used to write programs for heterogeneous platforms. OpenCL programs can use an address space qualifier (such as _ _ global for global variables or __local for local variables) when declaring a variable to specify the memory region where the object should be allocated. The OpenCL implementations for the GPU in the Ivy Bridge accesses memory through the GPU-specific L3 cache and the CPU and GPU shared Last Level Cache (LLC). Accesses to global variables go through the GPU L3 cache and the LLC. Accesses to local memory (also referred to as shared local memory because this local memory is shared by all work items in a workgroup) are allocated directly from the GPU L3 cache. Thus, the GPU L3 cache can be used as a scratch pad or as a cache. The size of this memory is 64KB (obtained using the standard "clGetDeviceInfo()" OpenCL call) for both platforms, the Desktop and the Ultrabook. The CPU does not have hardware support for local memory, so in principle codes running in the CPU do not benefit from using local memory. Additional details can be found in Int [2013b].

\subsection{Evaluation Methodology}

For the experimental results, we measure the time of thousands of iterations of the application and report the average. This is realistic for many vision applications, which are expected to perform analysis (e.g., detection) over a continuous input of frames, fed from the device camera. This setup is especially important for the Ivy Bridge GPUs, since the running times have high variance in the first few iterations but stabilize after some "warm-up" iterations. For all the experiments reported here, our input image size is 600 by 416 pixels.

For power and energy measurements, we use hardware energy counters available in the Ivy Bridge architecture [David et al. 2010]. They measure three domains: "package," "core," and "uncore." Package means the consumption of the whole chip, including the CPU, the GPU, memory controllers, and so forth. Core is the CPU domain and Uncore is the GPU domain. For power measurement of the whole system, we plug a power meter into the machine's power input.

The new Intel Turbo Boost Technology 2.0 [Rotem et al. 2012] makes the measurements complicated on this architecture. In a nutshell, it accumulates an "energy budget" during idle periods and uses it during burst activities. Thus, the processor can possibly go over the Thermal Design Power (TDP) for a while. It takes a few seconds for it to reach that limit and several seconds to go back to the TDP limit. This can change the performance and power of the processor significantly. One might turn this feature off for accurate measurements. However, it is an advanced strength of the architecture that can enhance the user experience significantly (e.g., for interactive use), so it should not be ignored. For our measurements, we run each program for around 10 seconds (which seems to be a valid common use case) and average the iteration times and power consumption.

We used the machine peak performance numbers reported in the Intel documentation. ${ }^{3}$ However, those values are computed using the maximum frequency value and AVX vector units, but, as mentioned, the processor cannot be at the maximum frequency for a long time. Thus, in many cases, peak performance numbers are upper bounds of the actual peak performance.

\section{OPTIMIZATION OF KERNELS IN OPENCL}

In this section, we describe the optimizations we applied to the OpenCL kernels described in Section 2.1. Then, in Section 3.4, we analyze the performance impact of each optimization. The OpenCL codes run in both the CPU and the GPU, but it is possible that an optimization that works well for the GPU would hurt the performance

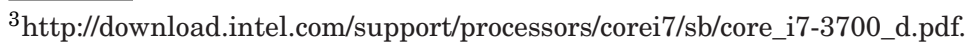




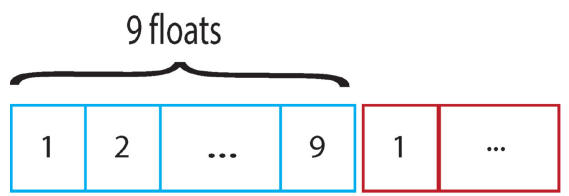

(a) original coefficient layout

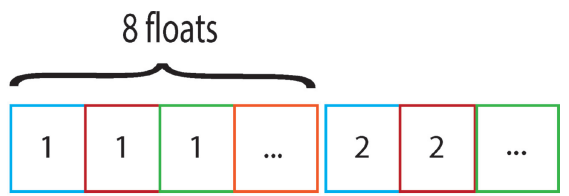

(b) vectorized coefficient layout

Fig. 1. Change of coefficient data layout for vectorization.

when running on the CPU or vice versa. From now on, we will refer to the blockwise distance kernel as filter, the cell histogram kernel as histogram, and the pairwise distance kernel as classifier.

\subsection{Filter Kernel}

Here, we describe the optimizations that we applied to the filtering algorithm shown in Figure 1.

3.1.1. Parallelism. We exploit parallelism by dividing the image across multiple workgroups with several work items. Then, each work item runs the 100 filters on its image block. We use a 16-by-16 workgroup size following Intel OpenCL SDK's recommendation (considering also our working set memory size). In addition, we use the Kernel Builder (from the Intel OpenCL SDK) tool's workgroup size auto-tuning capabilities to make sure this is the best size.

\subsubsection{Loop Unrolling. We completely unroll the inner loop, which has nine iterations.}

3.1.3. Vectorization. This transformation tries to exploit the CPU's AVX vector units. Without vectorization, this kernel calculates the response of every filter on a 3-by3 image patch, keeping track of the maximum one. This requires nine multiply-add operations, followed by an update guarded by an if statement. In this form, the inner loop cannot be fully vectorized. Since $A V X$ supports eight operations at a time, we can vectorize eight of the multiplies and partially vectorize the sum reduction, but we still need to run one sequentially. Thus, to enable efficient vectorization, instead of working on one filter at a time, one can consider eight of them at the same time. Note that the number of filters (100) is not a multiple of eight, so we need to handle the last four filters separately. Each pixel value needs to be replicated (broadcast) in a vector to participate in the vector operations.

This transformation needs a reorganization of the filter coefficients' data structure in the memory. Originally, a filter's nine coefficients are located in consecutive memory locations (Figure 1(a)). However, we need the first coefficients of eight filters to be together to be able to load them in an SIMD vector (Figure 1(b)). Figure 1 illustrates these layouts using different colors for different filters, and numbers for different elements of a filter. Thus, effectively, we are transposing each $8 \times 9$ submatrix of eight filter coefficients to a $9 \times 8$ one. This transformation is generally useful for vectorizing various filters of different sizes since the number of coefficients most probably does not match the SIMD size. Note that this transformation can be thought of as a customized instance of the Array of Structures (AoS) to Structure of Arrays (SoA) transformation.

3.1.4. Local Memory. This optimization is specific to the GPU. The filter kernel operates on an image and the 100-filter coefficients. The filter coefficients occupy 3.5KB that we copy (using all the work items in parallel) to the local memory. Each workgroup also copies the image block it needs. This optimization may hurt performance when the code runs on the CPU due to the copying overheads. This is evaluated in Section 3.4. 


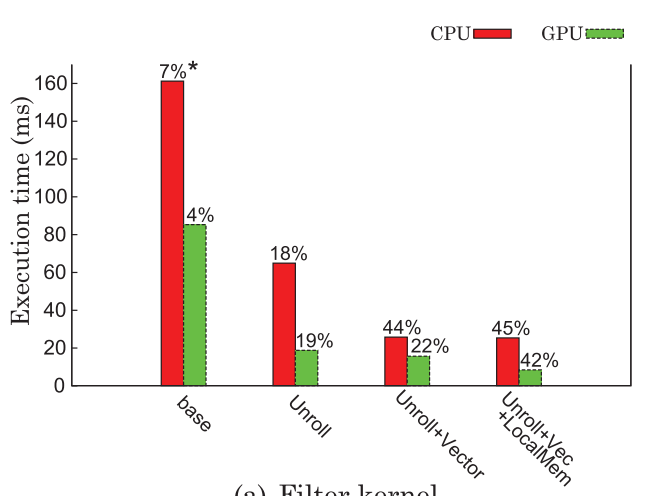

(a) Filter kernel

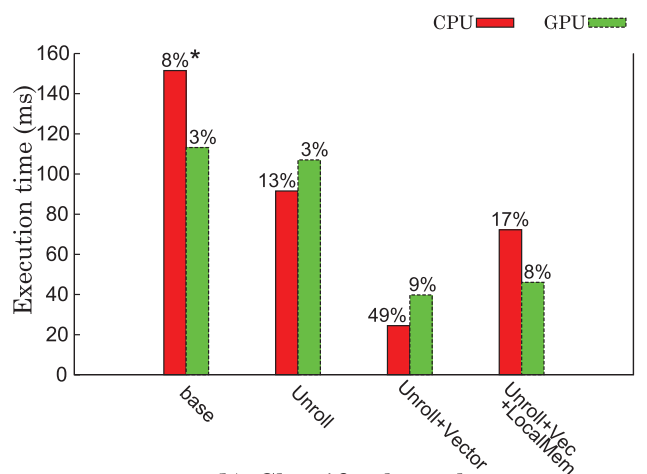

(b) Classifier kernel

Fig. 2. Execution time of kernels with different optimizations (on Ultrabook). * Percentage of peak performance.

\subsection{Cell Histogram Kernel}

The parallelism is achieved through a scatter operation. Every work item in a workgroup accumulates a subset of the values inside the image block to their respective histogram bins. Note that this is a potential race if two or more work items in the same workgroup try to increment the same histogram bin. This race can be avoided if the language and the hardware allow for "atomic_add" directives for floating point numbers. However, these atomic operations serialize memory accesses and can hurt the performance significantly.

We allow this race in our OpenCL kernel because our Monte Carlo simulations have shown that the probability of such a race is low given the distribution of filter indexes in natural image patches. Therefore, we do not expect the race conditions to change the shape of the histograms drastically, and we have validated this through experiments. Unlike scientific applications, media programs do not need full accuracy in many cases, and we should exploit this for better performance and energy efficiency.

\subsection{Classifier Kernel}

3.3.1. Parallelization. Parallelizing this code is similar to a tiled matrix multiply, where a workgroup is responsible for a tile of the output matrix (as with the filter, we use $16 \times 16$ tiles).

3.3.2. Loop Unrolling. We manually unroll the innermost loop, which has 16 iterations.

3.3.3. Vectorization. Vectorizing this code is easy as operations are done in an elementby-element fashion, with elements in consecutive memory locations. After accumulating differences in a vector, a sum reduction is required (which we implement as a dot product with an identity vector).

3.3.4. Local Memory. All the work items load the two blocks of elements they want to work on in parallel in the local memory.

\subsection{Performance Evaluation}

In this section, we evaluate the performance impact of each of the optimizations. Figure 2(a) shows the execution time for filter when running on the CPU or the GPU. The bars show the cumulative impact of the different transformations. Thus, Unroll+Vec+LocalMem corresponds to the execution time after all the optimizations have been applied. As Figure 2(a) shows, after applying all the aforementioned optimizations, this kernel runs more than 10 times faster on the GPU than the original 
Table III. Effective Optimizations for Filter and Classifier Kernels on Ultrabook

\begin{tabular}{|l||c|c|c||c|c|c||c|}
\hline \multicolumn{1}{|c||}{} & \multicolumn{3}{c||}{ GPU } & \multicolumn{3}{c||}{ CPU } & \multirow{2}{*}{ Same code? } \\
\cline { 2 - 7 } Kernel & Unroll & SIMD & Local-Mem & Unroll & SIMD & Local-Mem & yes \\
\hline filter & yes & yes & yes & yes & yes & no & yes \\
\hline classifier & yes & yes & no & yes & yes & no & yes \\
\hline
\end{tabular}

nonoptimized code. It now takes only $8.46 \mathrm{~ms}$. It also made it 6.4 times faster on the CPU (takes 25.5ms for the same image). Loop unrolling speeds up this kernel for both the CPU and the GPU. Vectorization speeds up filter for the CPU significantly. Also, even though the GPU does not have CPU-like vector units, execution times decreases by about $16 \%$ (this is discussed in Section 3.4.3). We also note that the use of the local memory for the filter coefficients does not have a significant overhead on the CPU. Thus, the same kernel code can be used for both architectures.

Figure 2(b) shows the results for the classifier. As the figure shows, both unroll and vectorization improve the performance significantly. However, the use of local memory degrades performance for both devices. Hence, we did not use the local memory for classifier and, again, the same code is used for both the CPU and the GPU.

To assess the utilization of the CPU and GPU, we measured the MFLOPs for both filter and classifier. Numbers on top of the bars in Figures 2(a) and 2(b) show the performance of each code as a percentage of the peak performance of the machine. Our numbers show that filter runs at $45 \%$ and $42 \%$ of the peak performance on the CPU and GPU, respectively. Classifier runs at $49 \%$ and $9 \%$ on the CPU and GPU, respectively. Thus, filter utilizes both the CPU and GPU very well, while classifier only has that level of utilization on the CPU. The inefficiency of GPUs for certain workloads has been discussed in related work [Lee et al. 2010]. However, 9\% utilization might be considered high for certain workloads on the GPU.

Note that a more optimized code usually results in faster and more energy-efficient execution. In fact, in our experiments, we observed that the programs were consuming the same or similar power before and after the optimization. Thus, decreasing execution time almost directly results in lower energy consumption in this case.

Table III summarizes our results about which optimizations were effective on each device. Note that the OpenCL filter kernel that we use in the following sections uses the local memory optimization, since it does not hurt performance, and this allows us to have a single-code version for both devices.

Next, we discuss the performance results, specifically the impact of using local memory, branching effects, and vectorization, in more detail.

3.4.1. Local Memory Usage. Our results in Figures 2(a) and 2(b) show that the use of local memory is important for the filter kernel but undesirable for the classifier.

Using the local memory is essential for the filter kernel on the GPU. The reason is that a small set of constant data (the filter coefficients) is needed for the whole execution (all the iterations of the outer loop). Relying on the GPU L3 cache is not effective because the data from the image that is being accessed at the same time might replace the filter coefficient in the cache.

On the other hand, using the local memory is detrimental for the classifier kernel on the GPU of our Ivy Bridge Ultrabook. However, using the local memory for the classifier improves the performance on the smaller GPU (HD Graphics 2500 device) of the Desktop platform by 35\%, even though the architecture is essentially the same (the Desktop GPU has only six CUs, while the Ultrabook GPU has 16 CUs).

To understand the differences in performance (in the absence of performance counters), we used the memory streaming microbenchmark of the uCLbench package [Thoman et al. 2011] that measures the effective bandwidth to memory. This 
benchmark allocates arrays in memory (either local or global), which are accessed by all the work items repeatedly. Our experimental results show that the effective bandwidth of local memory is less for the Ultrabook GPU than for the Desktop GPU (7.8GB/s for the Ultrabook vs. $10.3 \mathrm{~GB} / \mathrm{s}$ for the Desktop) when local arrays are accessed. On the other hand, the effective bandwidth of global memory is about the same for both machines $(7 \mathrm{~GB} / \mathrm{s}$ for the Ultrabook vs. $7.4 \mathrm{~GB} / \mathrm{s}$ for the Desktop) when global arrays are accessed. Notice that the working set of the classifier is just the data that we are placing on the local memory and fits in the $64 \mathrm{~KB}$ of the GPU L3 cache. Thus, since the Desktop has a higher effective bandwidth when accessing the data in the local memory, the local memory optimization reduces execution time. However, in the Ultrabook, the bandwidth is similar and the use of local memory introduces some copying overheads.

Using local memory for the code running on the CPU introduces some extra copying overhead. While this overhead is not visible for filter because of the small size of the filter coefficients' data structure, it adds a significant overhead to the classifier kernel, due to the larger size of the data structure allocated in local memory.

3.4.2. Loop Unrolling and Branch Overhead. Unrolling results in a significant performance improvement in both kernels, classifier and filter, for both the CPU and GPU. In the CPU, unrolling decreases loop overhead and increases instruction-level parallelism. In the GPU, unrolling reduces the number of branches.

Branches on the GPU can have a significant impact on performance, especially in the case of divergent branches where work items (threads) of a CU take different paths and each branch path has to be serialized. On the other side, nondivergent branches, where all the work items follow the same path, are usually fast.

To assess the impact of nondivergent branches on the Ivy Bridge integrated GPU, we modified the filter kernel and replaced the "if" condition that finds the maximum filter response with additions that sum the filter responses (notice that this branch, although data dependent, is mostly nondivergent, as work items execute on neighboring pixels that tend to be similar and hence the maximum response filter is mostly the same for all the work items). This change made this code run $13 \%$ faster on the integrated GPU. We also ran both codes (with and without the "if" statements) on the Fermi Nvidia GPU and found that the code without the branches had only $3 \%$ improvement. In addition, we used the "branch overhead" benchmark of the uCLbench package [Thoman et al. 2011] to assess the difference in performance between divergent and nondivergent branches. In this benchmark, different cases of branch divergence are compared. For example, a branch might be taken by all the work items, a subset of them, or only one. The experimental results show that the Ivy Bridge's integrated GPU is performing much better for nondivergent branches, as benchmarks can be up to 10 times slower on the Ivy Bridge's integrated GPU when branches are divergent.

Overall, our experiments show that nondivergent branches have a higher effect on the Ivy Bridge GPU than on a Fermi GPU. Thus, loop unrolling (that removes nondivergent branches) is an important optimization for this platform. Other nondivergent branches, such as the "if" associated with the max operator, cannot be removed with loop unrolling and would benefit from better hardware support for nondivergent branches.

3.4.3. Vectorization. Vectorization speeds up both the codes for the CPU, as it makes it easier for the compiler to generate code using the AVX vector extensions in the Ivy Bridge. When running on the GPU, the classifier is about 2.8 times faster with vectorization, despite the fact that vector units need to be emulated on the GPU, which might have some overheads. One reason is that the vector code has more unrolling on the GPU implicitly. Thus, to assess the effect of further unrolling, we unroll the nonvectorized code's outer loop as much as it is beneficial (and "jam" it into the inner 
loop, which is already unrolled). This code runs faster, but still 1.8 times slower than the SIMD version. The other reason for the difference in performance is found by looking at the code generated by the compiler for both versions (with and without SIMD). For the code with SIMD, the compiler generates different memory load instructions with better alignment, which is important for performance.

As mentioned, the filter kernel runs only slightly (13\%) faster on the GPU when vectorization is applied.

\section{COMPARISON WITH OTHER PROGRAMMING PARADIGMS}

In this section, we assess if OpenCL is a suitable paradigm for the CPU, since it is desirable to have a single programming paradigm for both types of devices.

For that, we compare the programming effort and execution times of the OpenCL filter code versus implementations of the same code written with other programming models for the CPU. Filter code is chosen for the comparison because it is a computeintensive kernel, based on a convolution operation used by many computer vision applications.

We run the experiments of this section on the Desktop's CPU, since it is more powerful and will reflect the effects better. In addition, the Ultrabook's CPU does not support SSE vector instructions. Note that for all the experiments, we use 4-byte "float" precision numbers (which are enough for the filter kernel).

\subsection{OpenMP with Compiler Vectorization}

Since OpenMP is well suited to exploit data-parallel computation in multicores, we compare the OpenCL code with an OpenMP implementation. Although one could expect perfect speedups, our results show an overhead of $8 \%$ with respect to perfect scaling. This is due to the overhead of spawning and joining threads for every loop invocation on a different image.

To exploit the machine's potential, we need to exploit the CPU's vector units. The simplest way is to have the compiler do this task. The Intel compiler that we use (Section 2.5) can vectorize this code but needs the "/fp:fast" flag to enable optimizations that can cause minor precision loss in vectorization of reductions. In addition, by looking at the assembly code, we realized that it did not generate aligned loads, which was fixed by using the Intel compiler intrinsic function (__assume_aligned()).

Furthermore, with the hope that the compiler would generate better code, we generated another code version where we applied, at the source level, the transformation we applied to vectorize the OpenCL filter kernel (Section 3.1).

\subsection{OpenMP with Manual Vectorization}

We vectorized the code manually using vector intrinsics that map directly to assembly instructions. A disadvantage of this approach is that the code is not portable as it is tied to a specific machine's instruction set and a compiler. Furthermore, it is close to the assembly level and hence, the programming effort including code readability and debugging will suffer. Nonetheless, if the performance difference can be very high, one might prefer paying the cost. We wrote three versions: using AVX and SSE, using only SSE, and using only AVX.

4.2.1. $A V X+S S E$. The Ivy Bridge architecture supports the AVX and SSE instruction sets. AVX instructions can work on eight floating point elements, while SSE ones can only handle four elements. We use SSE, since AVX does not have an instruction equivalent to SSE's “_mm_comigt_ss” (that compares two values and returns a 1 or a 0 depending on which one is larger), which simplifies the coding. Thus, we use AVX for multiply and add operations and SSE for conditional comparisons. Note that mixing 
AVX and SSE instructions can have significant translation penalties on Ivy Bridge [avx 2011]. However, we use the "/Qxavx" flag to ask the compiler to generate AVX counterparts whenever possible. In addition, we use the Intel vTune Amplifier to make sure these penalties are avoided. Since this kernel needs to find which filter resulted in the maximum response value, we compare the max_response against each response value. A sample comparison is shown here, where we permute the result vector and compare the lowest-index element using the "_mm_comigt_ss" intrinsic:

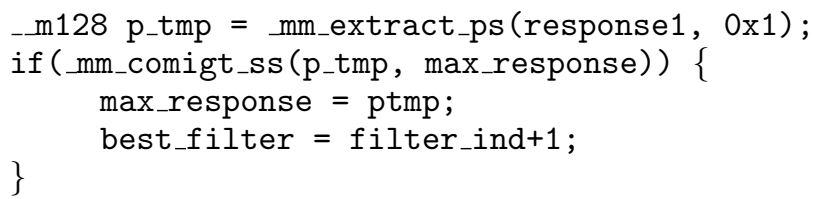

Note that we provide code snippets to be able to compare the complexity of different methods. We refer the interested reader to Intel's documentations to fully understand the details.

4.2.2. SSE. We implemented an SSE version to evaluate AVX versus SSE and measure the effect on performance of SIMD width.

4.2.3. AVX. We also implemented a version that only uses AVX instructions. The implementation compares all the responses in parallel, gathers the sign bits in an integer mask, and examines each bit separately. If the maximum response needs to be updated, we use a permutation instruction to broadcast the new maximum to the register, repeat the comparison, and update the integer mask. There is a small complication because of “_mm256_permute_ps" instruction's semantics. Since it can only choose from each fourelement half of the register separately, we need to consider each half of the responses separately and copy it to the other one. Thus, the initialization code for comparing four elements of responses is shown here:

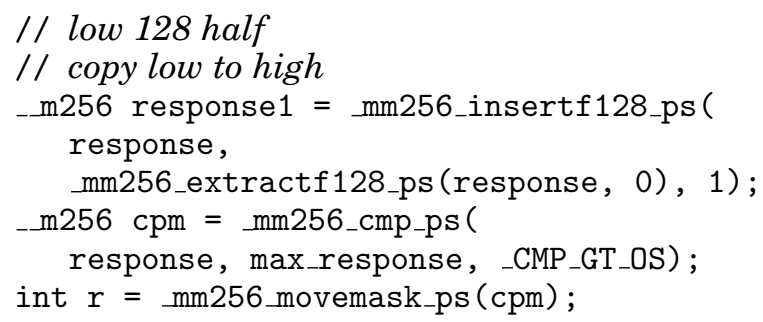

After that, we will have four tests of the mask with possible updates similar to this one:

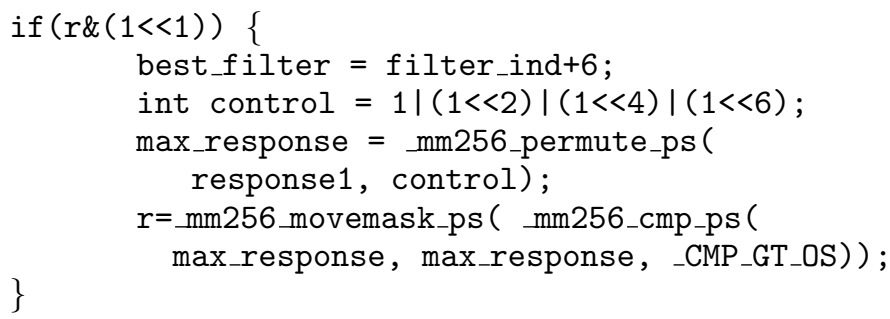




\subsection{OpenCV Library Calls}

OpenCV [Bradski 2000] is an open-source library consisting of many low-level image processing algorithms, as well as many high-level algorithms frequently used in computer vision. It is by far the most utilized common code base for vision research and applications. We constructed the object detection algorithm using standard library data structures and function calls to OpenCV in order to compare what is achievable in terms of performance using the standard $\mathrm{C}++$ interface. We link against the standard distribution of the OpenCV binaries, which is not multithreaded. ${ }^{4}$

The filter kernel can be constructed simply by 100 calls to the OpenCV twodimensional filtering function. Between each OpenCV filtering function call, we do a pass over the output array to determine if the current filter response value is higher than the maximum value observed and replace the assignments in the output array accordingly. The classifier kernel can be simply constructed by taking the norm of the differences for every pair of rows in the two input matrices. Row isolation, vector differencing, and vector norms are all standard library calls in OpenCV. The histogram kernel is not achievable through standard library calls, but the ViVid call can be used on standard OpenCV data types with minimal changes.

Our experimental results show that the OpenCV implementation of the filter kernel runs 15 times slower than the OpenCL version of ViVid running on the CPU of the Ultrabook. Notice that our OpenCL code runs in parallel (two cores of the Ultrabook), while the OpenCV code runs sequential. Also, the OpenCV code that we use has hardcoded SSE2 intrinsics (we verified by looking at the library code), while our OpenCL code uses the AVX vector instructions (when running on the CPU). However, these two points still do not justify the big difference in performance. This substantial difference in performance appears because the OpenCV code does not take advantage of locality, as we need to reload the image 100 times. In our OpenCL code, each image pixel is loaded only once, as the 100 filters are applied to each pixel before moving to the next one. Notice that experimental results for classifier show that the OpenCV code is also significantly slower than our OpenCL code. The reason is that while the OpenCV library has an efficient matrix multiplication call, what we need is a customized operator (the square of the two values' differences or Euclidean distance), which needs to be realized in an inefficient manner (as mentioned earlier).

While the code using the library calls is very concise and straightforward, the nonperfect adoption of OpenCV library primitives for our algorithm results in performance degradation. This is because each library call has some overhead, and no optimization is possible across library primitives. Vision applications can benefit significantly from specific low-level optimizations based on the expected input and output structures, as well as computational patterns of individual kernels. Thus, current vision libraries are unable to solve the entire parallel programming problem for vision applications, as the resulting code is not fast enough for production use. However, ease of use has made these libraries, such as OpenCV, very good candidates for application prototyping and development.

\subsection{Performance and Effort Comparison}

4.4.1. Performance Comparison. Figure 3 shows the execution time and the percentage of peak performance obtained by different schemes running on the Desktop platform (results in the Ultrabook are similar as these experiments are mainly concerned with vectorization). In the schemes evaluated, orig-novec corresponds to the baseline OpenMP code where compiler vectorization is disabled; orig-auto corresponds to the

\footnotetext{
${ }^{4}$ Some OpenCV functions can be made multithreaded by linking against Intel Thread Building Blocks [Reinders 2007].
} 


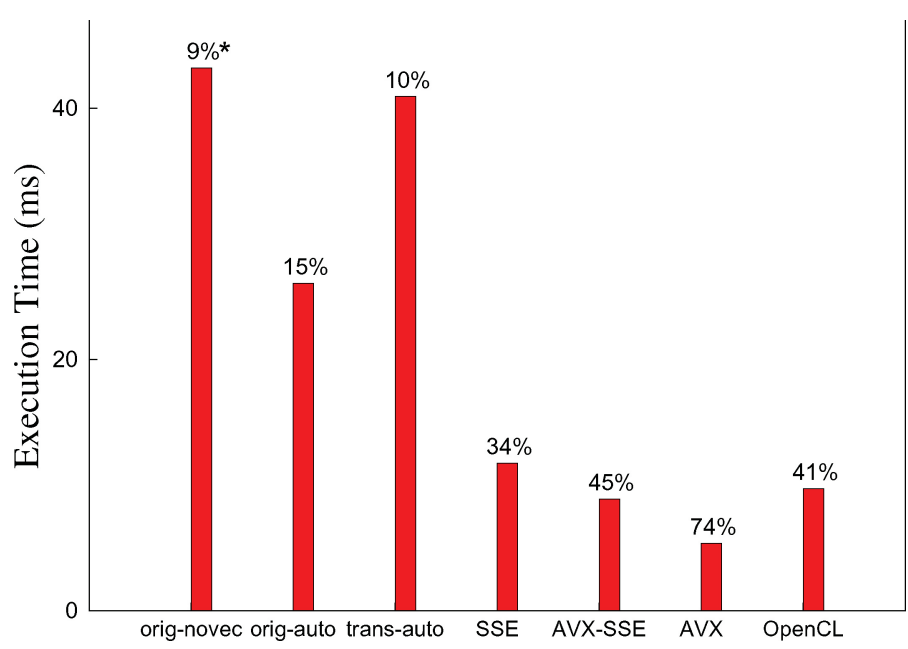

Fig. 3. Performance comparison of filter kernel in different paradigms (on Desktop). * Percentage of peak performance.

OpenMP code autovectorized by the compiler; trans-auto is the OpenMP code transformed for vectorization at the source level (as in the OpenCL code in Section 3.1) and automatically vectorized by the compiler; the three-code versions using intrinsics are labeled $S S E, A V X+S S E$, and $A V X$; and OpenCL corresponds to the OpenCL code optimized as discussed in Section 3.1.

As the figure shows, the autovectorized codes (orig-auto and trans-auto) run significantly slower than the OpenCL code $(26.06 \mathrm{~ms}$ and $40.93 \mathrm{~ms}$ versus the $9.74 \mathrm{~ms}$ time of the OpenCL code). Checking the generated assembly code of orig-auto, we see that the compiler has generated vector code, but it is not as efficient as our manual code since it could not perform our transformation automatically (which is expected). The figure also shows that the performance of trans-auto improves only slightly with respect to orig-novec, because, although simple, the compiler cannot analyze the transformed code. Thus, autovectorization, although easy to use, is not a good solution in terms of performance, even for the simple loops of our filter kernel.

With respect to the vector codes using intrinsics, $A V X$ is the fastest code and is $46 \%$ faster than the OpenCL code. As expected, $S S E$ is the slowest of the codes using intrinsics, due to the shorter vector units. However, the SSE code is the shortest, as the number of filters (100) is now an exact multiple of the SIMD width (four elements). Thus, wider SIMD units increase the overheads of handling boundaries. Finally, the $A V X+S S E$ is only $8 \%$ faster than the OpenCL counterpart.

For comparison purposes, we implemented, using AVX, a filtering kernel without branches and comparisons (that does not find the index of the best filter). It uses a small "reduction tree" method to perform fewer SIMD max operations to find the maximum from the eight responses. Our $A V X$ version is just 5\% slower than this one, showing that we have alleviated most of the comparison overheads by comparing in parallel and pushing other instructions inside the "if" statements.

4.4.2. Programming Effort Comparison. To quantify the programming effort of each paradigm, we use the Halstead's productivity metrics [Halstead 1977; Weyuker 1988; González and Fraguela 2010]. In Halstead's Elements Software Science [Halstead 1977], a program is considered as a string of tokens, which can either be operands or operators. The operands are either constants or variables, while the operators are 
Table IV. Software Metrics for Different Implementations of the Filter Kernel

\begin{tabular}{|l|c|c|c|c|c|c|c|}
\hline Paradigm & $\eta_{1}$ & $\eta_{2}$ & $N_{1}$ & $N_{2}$ & $V$ & $E$ & SLOC \\
\hline orig-auto & 23 & 61 & 230 & 214 & 2838 & 114504 & 68 \\
\hline SSE & 23 & 102 & 494 & 467 & 6694 & 352458 & 133 \\
\hline AVX-SSE & 23 & 101 & 682 & 645 & 9228 & 677725 & 187 \\
\hline AVX & 24 & 106 & 903 & 836 & 12211 & 1155752 & 212 \\
\hline OpenCL & 22 & 99 & 691 & 625 & 9105 & 632307 & 162 \\
\hline OpenCV & 12 & 25 & 59 & 63 & 635 & 9609 & 15 \\
\hline
\end{tabular}

symbols or their combination that can affect the value or ordering of operands. Let $\eta_{1}$ be the number of unique operators, $\eta_{2}$ the number of unique operands, $N_{1}$ the number of occurrences of operators, and $N_{2}$ the number of occurrences of operands. Derived metrics that can be used to quantify programming complexity are defined as follows:

Program Volume: $V=(N 1+N 2) \log _{2}\left(\eta_{1}+\eta_{2}\right)$

Program Difficulty: $D=\frac{1}{2} \frac{\eta_{1} N_{2}}{\eta_{2}}$

Programming Effort: $E=D V$

Table IV shows these metrics for different implementations of the filter kernel. The last column of Table IV shows the number of Source Lines of Code (SLOC). The table shows that performance is correlated with effort; higher performance requires more programming effort, which is to be expected. $A V X$ has the highest performance and effort, while compiler autovectorization has the least of both. From these numbers, OpenCL Programming Effort and Program Volume metrics are similar to those of AVX-SSE; both also deliver similar performance. The table shows that $\eta_{1}$ is almost the same for all the code versions, while $\eta_{2}$ is 61 for orig-auto and around 100 for all the others. These additional variables appear as a consequence of unrolling, which has been applied to all code versions but orig-auto. N1 and N2 are also larger in AVX because it needs some code to handle the leftovers after loop unrolling. In addition, the code to compute the index of the filter with the maximum response is more complex, as described in Section 4.2.3.

Notice that all these metrics do not fully capture the complexity of each code. They are based on the number of operators and operands but do not take into account the complexity of each operator. For example, addition is much simpler than a vector intrinsic function of AVX. Thus, these metrics may be highly optimistic for the vector implementations.

Overall, OpenCL provides a good balance in programming effort and performance. It is much faster than the autovectorized versions and it is close to the low-level intrinsics versions. It is 1.8 times slower than the $A V X$ code with "ninja optimizations," but the effort is significantly less (1.8 times less Halstead Effort). Therefore, programming in OpenCL is effective for the CPU as well as the GPU, and bringing the GPU on the die did not impose significant programming effort (since the same code runs on the CPU as well). Thus, OpenCL has the advantage that a single programming model can be used to run in both the CPU and GPU. It is possible that the code versions that run the fastest will be different among platforms, but the programming effort does not increase significantly, because the different versions need to be tried in both platforms in the optimization and tuning process anyway.

Note that we do not claim OpenCL is performance portable across platforms in general. We believe that given the data-parallel nature of vision algorithms, in many cases, the same baseline algorithm can be written for the CPU and GPU in OpenCL. However, tuning transformations need to be evaluated separately for each device. For this study, our target of the OpenCL tuning was the GPU, but the experimental results 
show that the transformations also worked for the CPU, resulting in the same kernel codes.

\subsection{Possible Hardware and Software Improvements}

Vision and video analytics (and their filtering kernels) are important applications for heterogeneous on-chip architectures. Thus, we list a set of possible improvements to the hardware and system software that vendors might consider for this class of applications.

The first one is related to the algorithms that the compiler can recognize and vectorize automatically. We observed that neither the Intel compiler nor the OpenCL compiler can generate efficient vector code for the max reduction (and finding the index corresponding to max) used in the filter kernel. When we examined the assembly code, we found out that the OpenCL compiler generates permutations and comparisons similar to our $A V X+S S E$ version. However, the compiler should be able to automatically generate more efficient code [Ren et al. 2006; Maleki et al. 2011], following a similar approach to the one in the $A V X$ code evaluated in Figure 3.

The second one deals with a common operation in these types of kernels. We have observed that multiply and add operations are used together extensively. Thus, FusedMultiply-Add can improve the performance significantly. The "FMA" vector extension addresses this point, which is available in some new processors (such as the ones using Intel Haswell microarchitecture).

Our transformation optimizes filter kernels significantly, but they could become even faster with more hardware support. Finding the maximum and corresponding index in a vector is a reduction across the elements of a single SIMD vector, or a horizontal max operation (in Intel's terminology). In current SSE and AVX standards, there are a few horizontal operations, such as an addition that just reduces two adjacent elements. This could be further extended to perform a full reduction, which will improve multimedia applications in general [Corbal et al. 2001; Talla et al. 2003; Bik et al. 2006]. In fact, to estimate how much improvement we can achieve with a reduction instruction, we replaced the instructions to find the maximum response in our $A V X$ kernel with just a horizontal add instruction. This improved the performance by more than $34 \%$. Thus, more targeted hardware support can lead to significant improvements in future machines.

\section{APPLICATION PERFORMANCE AND ENERGY}

This section evaluates and analyzes the execution time and energy consumption of the kernels described in Section 2.1 and optimized in OpenCL in Section 3. We also evaluate and analyze different mappings for our application on the CPU and GPU for better performance and energy efficiency.

Figure 4(a) shows the execution time of each kernel on the CPU and GPU. full-app shows the results for all three kernels running on either the CPU or the GPU. The GPU is about three times faster for the filter kernel and 2.3 times faster for the histogram one. However, it is more than 1.3 times slower for the classifier kernel. Note that classifier performs fewer floating point operations per data element (see related work for analysis of data-parallel kernels on the CPU and GPU [Lee et al. 2010]). For the full application, the GPU is slightly faster (less than 8\%) than the CPU.

Figure 4(b) shows the power consumption of the processor for each individual kernel and for the full application running on either the CPU or the GPU. Each setting is labeled after the running code and the architecture it is using. For instance, "class-G" means that the classifier kernel is running on the GPU. Each setting has three power consumption bars. We also show power numbers in the idle state. The red (left) bar is the power consumption of the whole processor chip (CPU, GPU, memory controller, 


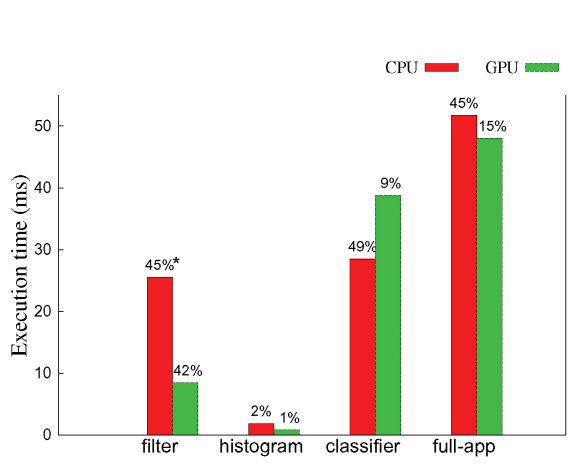

(a) Execution time

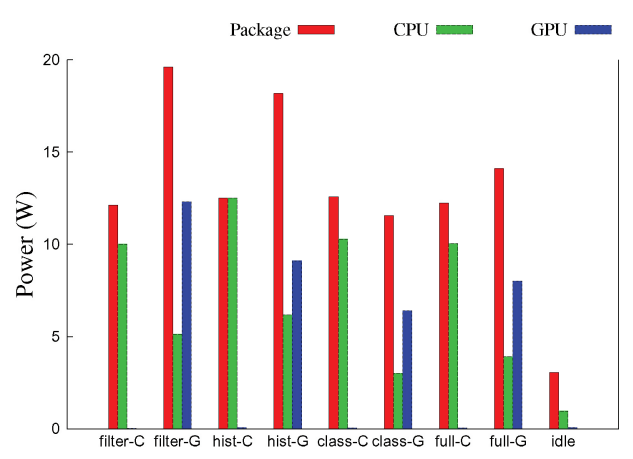

(b) Power consumption

Fig. 4. E xecution time and power consumption of kernels (on Ultrabook). * Percentage of peak performance.

etc.), while the green (middle) bar is just the CPU's consumption and the blue (right) one is just the GPU's. Note that we report the average power consumption over a period of execution (see Section 2.6).

We mostly consider the power consumption of the whole package (the red bar), as it corresponds to the cost one would pay. However, the power breakdown can give insights about some important aspects of the system. For instance, when the code is running just on the GPU, the CPU is still consuming considerable power. The reason is that the CPU and the ring interconnect are in the same voltage and frequency domain [Rotem et al. 2012] and the interconnect cannot be idled, since the GPU needs to connect to the LLC. Addressing this issue may lead to significant savings in power consumption when the application is only using the GPU. The reason is that, for instance, the CPU domain consumes $3 \mathrm{~W}$ (with probably a notable part contributed by the cores) from the $11.5 \mathrm{~W}$ total package power when the classifier is running on the GPU. On the other side, it consumes only $0.7 \mathrm{~W}$ in the idle state.

As shown in Figure 4(b), the GPU consumes more power than the CPU in all cases (e.g., comparing left bars of filter-G and filter-C) except classifier, which is not unexpected since the GPU has higher peak performance as well (see Table I). However, the GPU's power consumption varies depending on the workload. For instance, the classifier consumes around 11.6W, while the filter consumes about $18.2 \mathrm{~W}$ (around $36 \%$ difference). This is because the filter keeps the GPU almost fully occupied, while the classifier does not have full utilization. On the other hand, the CPU's consumption has less than $0.5 \mathrm{~W}$ variation across the board, even with its complex architecture and power management schemes. The reason is that all the kernels can keep it occupied, partly because of its adapting architecture and partly because it is not very powerful.

So far, we have seen that the GPU is faster but consumes more power. Since one factor is in favor of energy but the other is against it, we need to look at the energy metric. Figure 5 compares the energy consumption of the different kernels and full application on the CPU and the GPU. For the full application, the package consumes slightly more energy when running on the GPU (less than 7\%), while it varies across different kernels. If we look at the package consumption, the GPU consumes 36\% less energy for the histogram kernel and $46 \%$ less for the filter kernel. However, it consumes $20 \%$ more energy than the CPU for the classifier kernel. This, as pointed out by others, contradicts the general belief that the GPU architecture is more energy efficient for every highly parallel kernel [Lee et al. 2010]. The net energy is in favor of the CPU, since the classifier kernel is time consuming on the GPU.

In a nutshell, running the three kernels in ViVid on the integrated GPU of the Ivy Bridge Ultrabook is faster but consumes more power and energy. On our Desktop 


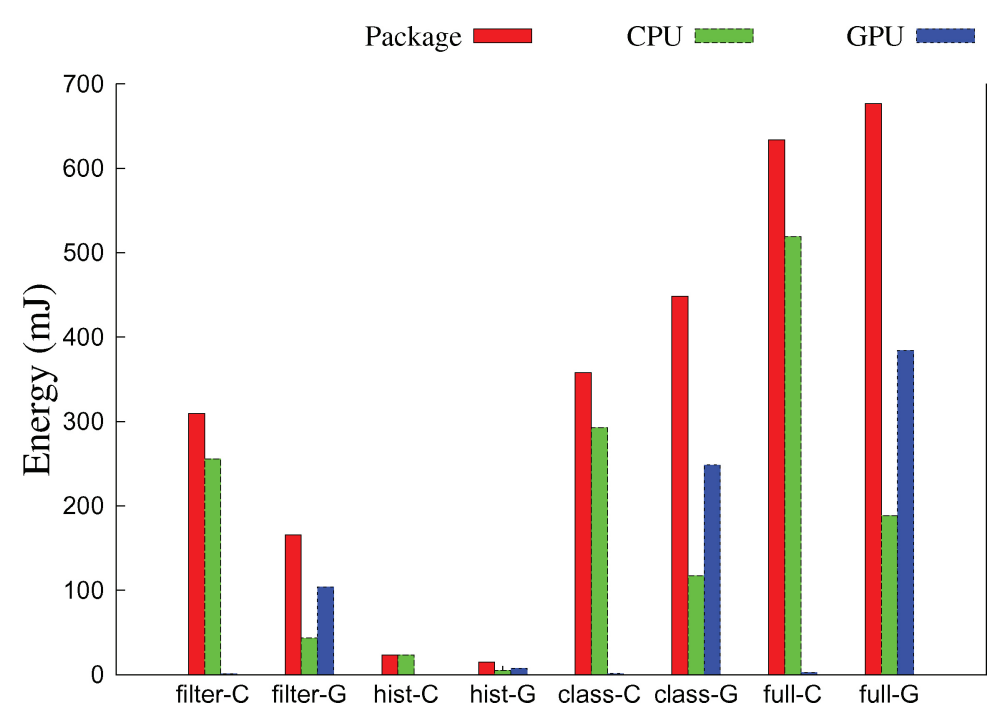

Fig. 5. Energy consumption (on Ultrabook).

system, with the same input size, the GPU is about five times slower and three times less energy efficient than the CPU for full-app, because it is small (so not very powerful in computation) but keeps the resources of the system busy. Thus, the balance of the system needs to be considered for portable devices that run vision applications. Comparing across platforms (but the same processor type), the CPU of the Desktop machine is about 2.5 times faster than the Ultrabook one for the full-app, but $19 \%$ less energy efficient.

\subsection{Mapping Strategies}

After understanding the different tradeoffs between the GPU and CPU for each kernel, the natural question is how to utilize the heterogeneous system for an application to achieve better performance and energy efficiency. Other than just running the code only on the CPU or the GPU, one could also try to map different kernels to the device where they run more efficiently. Figures 6(a), 6(b), and 6(c) show the execution time, power, and energy of different approaches, respectively. $C P U$ and $G P U$ correspond to running all the kernels in the CPU or in the GPU. Specialized corresponds to an execution where the filter and histogram are mapped to the GPU (where they run faster and more energy efficiently), whereas the classifier is mapped to the CPU (where it is faster and more energy efficient). In specialized, when the GPU is executing filter or histogram, the CPU is idle, and vice versa (when the CPU runs the classifier, the GPU is idle). Overlap corresponds to an execution similar to software pipelining. It can be applied to streaming applications where parallelism can be exploited across multiple input images or frames, like multiple frames of a video. When using overlap, filter and histogram form the first stage of the pipeline operating on a frame in the GPU, while classifier is the second stage of the pipeline running on the CPU and operating on the GPU's results. Note that with overlap, since the CPU's work takes around three times longer than the GPU, the GPU will be idle for about two-thirds of the execution time. Note that the strategies so far will underutilize either the CPU or the GPU because of data dependencies. Therefore, one could split the image between the CPU and the GPU for maximum utilization, shown as split in the figures. Since the execution 


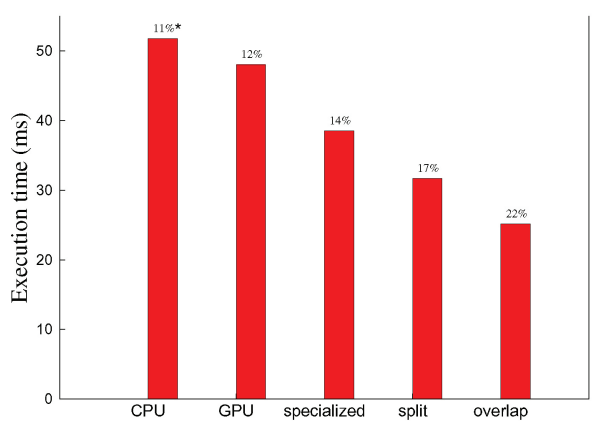

(a) Execution time

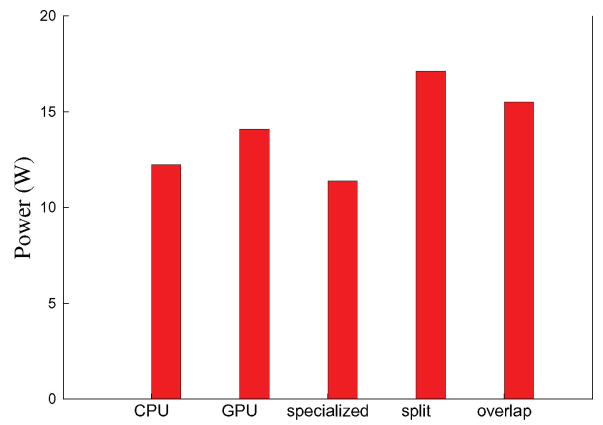

(b) Power consumption

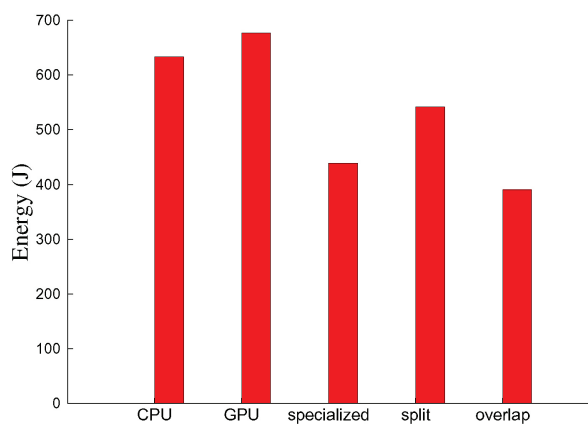

(c) Energy consumption

Fig. 6. Running full application on the CPU or GPU or utilizing both using different approaches. * Percentage of peak performance.

time of the application is almost the same for the CPU or the GPU, we split the image in half for our experiments.

When analyzing these strategies, one needs to keep in mind that this architecture has a dynamic power management scheme (Intel Turbo Boost 2.0 technology). It determines a fixed power budget at each time based on the temperature and assigns frequencies to the CPU and the GPU accordingly [Rotem et al. 2012]. Thus, for example, the CPU and the GPU are slower when they are running together as opposed to when the other is idle.

Figure 6(a) shows the execution time of different strategies for the full application. Specialized is more than $25 \%$ faster than just running on the CPU (20\% faster than the GPU), as one would expect. Split is about $39 \%$ faster than CPU, but it could be up to twice faster if the system did not have dynamic power management. Overlap obtains the best performance by running the kernels on the best type of processor but trying to keep them busier by software pipelining. It should be noted that, for our Desktop system, split did not result in any performance improvement compared to CPU. This is because the GPU is much slower (five times slower than the CPU) and the overheads of using it dominate. Thus, the balance of the heterogeneous systems seems important for these applications.

Figure 6(b) illustrates the power consumption of different strategies. Specialized has the least consumption, while split consumes the most. As one expects, overlap consumes more than specialized but less than split, because its resource utilization is in between the two. Note that power consumption does not necessarily correspond to execution speed here. 
Figure 6(c) shows the energy consumption of each strategy for an input image. specialized and overlap consume the least energy because they run each kernel where it runs the best. On the other hand, using only the CPU or the GPU is not energy efficient. Note that split is a very fast method, but it consumes much more power also, so it is not the most energy efficient in the end. specialized and overlap are $35 \%$ and $42 \%$ more energy efficient than the GPU-only method, respectively. They are also $19 \%$ and $28 \%$ more energy efficient than split, respectively.

Summary. Overall, our results show that to minimize energy consumption in these heterogeneous devices, one should try to exploit parallelism across devices, and each kernel should be mapped to the device where it is more energy efficient. Execution time should not be the only factor used to determine how to map an application, because the different devices have different power consumptions, resulting in different overall energy (e.g., specialized is slower than split, but more energy efficient). Thus, our overlap approach where parallelism is achieved through software pipelining seems the best strategy for these type of on-chip heterogeneous architectures. However, a couple of points need to be considered when choosing this strategy. First, it is desirable to have pipeline stages with similar execution times, as the execution time of this scheme is determined by the execution time of the longest kernel. Note that our application's stages do not have similar execution times, but this strategy is still the best. Second, this approach requires having more on-the-fly data. In our case, since the pipeline only has two stages, we have two frames on the fly (as opposed to one). In addition, since kernels execute in different devices, the frames need to move from device to device (in contrast with the split mechanism, where the data always stay in the same device). Since most of the vision applications (including ours) are very compute intensive, data movement usually is amortized easily by the numerous computations required per data element.

Our performance is superior or similar to recent works using much more capable discrete GPUs [Prisacariu and Reid 2009; Beleznai et al. 2011; Zhang and Nevatia 2008]. However, notice that real-time vision applications need to run at a certain number of frames per second. For instance, we can run at around 40 frames per second (fps) with overlap and 31fps with split, while 10fps might be enough for many object detection purposes. Applications requiring real-time object detection (33fps) can use the OpenCL code on this architecture. The extra available computation power can be used for more analysis or for other applications (e.g., if vision is only the interface for some other purpose). Note that when maximum performance is required (e.g., needed fps cannot be reached), one might need to trade energy efficiency for performance (e.g., specialized versus split, when overlap cannot be used).

One might need more compute power for future applications. Our experiments show that scaling the number of the GPU's CUs is effective. As noted in Subsection 2.5, the Ultrabook's GPU has 16 CUs, while the Desktop's GPU has 6 CUs, with similar architecture and frequencies. For all the kernels (as well as the full application), we see more than twice the speedup on the Ultrabook one, which supports the scalability of the architecture for these applications.

\subsection{Saving Energy with DVFS}

We saw that we can reach a detection rate that is more than enough for many applications. Thus, one might consider dynamic voltage frequency scaling (DVFS) for saving energy. However, the Ivy Bridge processor's DVFS does not seem to be effective for these compute-intensive codes. We applied DVFS to our application, and we could only save at most $5 \%$ of the energy while sacrificing $9 \%$ performance. The reason is that it makes the runtime so much longer (for compute-intensive codes) that it offsets the 


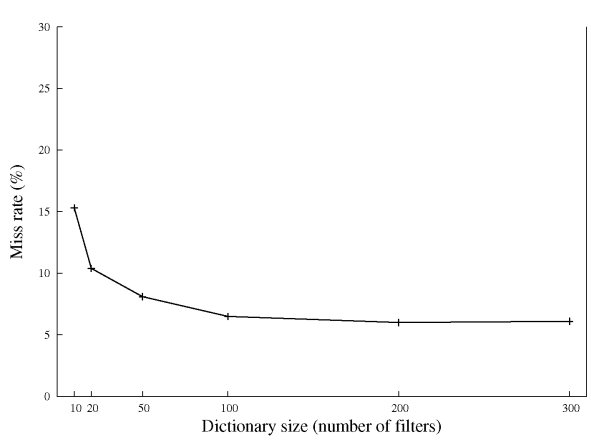

(a) Object detection miss rate

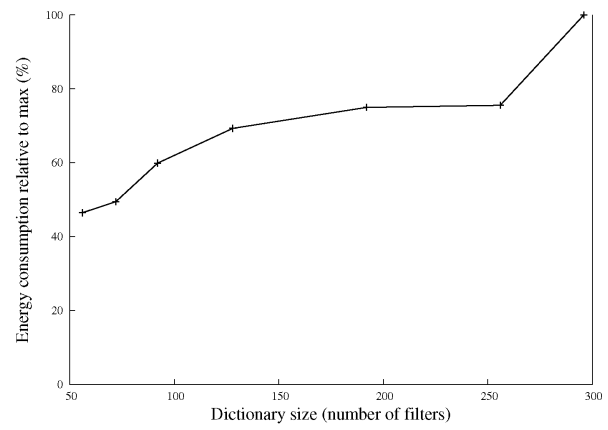

(b) Relative energy consumption

Fig. 7. Accuracy vs. energy consumption.

power savings. Thus, running the application for a while and then idling the processor seems to be the best solution for saving energy. In this case, savings will depend on sleep and wakeup latencies of the processor in the specificsxst usage.

However, we expect DVFS support to improve significantly in future devices, as vendors consider it in earlier steps of the processor design. For instance, when nearthreshold voltage (NTV) processors become available, DVFS will save much more energy [Dreslinski et al. 2010]. This will be very important for energy efficiency of many vision applications similar to ours.

\subsection{Trading Accuracy for Energy}

The visual descriptor we use is based on a model where the appearance of each $3 \times 3$ patch is characterized by finding its closest neighbors in a predetermined dictionary of $3 \times 3$ patch templates (filters). Naturally, larger dictionaries can capture wider varieties in appearances of patches. In the case of detection problems, this results in an increased modeling power for discriminating the appearance of the objects of interest versus the appearance of all other structures in natural images. However, as the size of the dictionary grows, more training samples are necessary to fully utilize the dictionary's modeling potential. Thus, for a given dataset, one can expect the detection performance to saturate at a large enough dictionary size, which we observe at around 150- to 200-item dictionaries in our example application. Figure 7(a) shows the miss rate of our object detection algorithm as a function of dictionary size (from previous work [Dikmen et al. 2012]). We chose 100 filters (dictionary size of 100) in this article because it provides enough accuracy for most applications [Dikmen et al. 2012].

However, since energy is a major constraint in portable devices, one might want to trade some accuracy for energy savings when the battery charge is low. In our application, accuracy is determined by the dictionary size (number of filters) as mentioned. Furthermore, the work of the algorithm also depends on the dictionary size. Thus, the number of filters might be a "knob" for the system to save energy according to the energy status of the device (battery charge) at runtime.

Figure $7(\mathrm{~b})$ shows the relative energy consumption when using different dictionary sizes. From this figure and Figure 7(a), one can conclude that we can save approximately $20 \%$ energy by going from 100 filters to 70 filters, which increases the miss rate only by around 1\%. This is a good tradeoff of energy and accuracy for many situations.

In a nutshell, we have considered four different techniques for better energy efficiency: (1) item program optimization, (2) Heterogeneity, (3) DVFS, and (4) decreasing accuracy. 


\section{RELATED WORK}

Previous works have shown that the use of heterogeneous architectures can improve performance and decrease energy consumption [Kumar et al. 2005]. In addition, mapping algorithms have been studied for heterogeneous systems [Luk et al. 2009; Liu et al. 2012; Jiménez et al. 2009; Ma et al. 2012]. Other forms of heterogeneity, such as off-loading virtual machine services (e.g., garbage collection) to smaller cores [Cao et al. 2012], have also been studied. However, the focus is mostly on mapping across different applications. In addition, integrated GPUs have not been considered.

A few programming paradigms such as OmpSs [Planas et al. 2013] or starPU [Augonnet et al. 2011] provide a unified programming paradigm for the CPU and the GPU and automatically perform load balancing and move the data as needed between the different nodes and GPUs. We restricted ourselves to OpenCL, since it is the only one supported by the integrated GPU in the Ivy Bridge machines. In addition, these works focus on programmability and performance, but not energy efficiency. Also, they focus on large systems rather than on-chip heterogeneous systems. Moreover, they do not focus on pipeline applications. Furthermore, as we have shown, automatic vectorization does not achieve high performance in our case.

The new architectures with on-chip GPUs are becoming increasingly more popular in the industry. These platforms include Intel's Ivy Bridge [Damaraju et al. 2012], AMD APU [Foley et al. 2012], and NVIDIA Tegra 250 [NVIDIA 2011]. Evaluation studies also show their advantages in performance and energy efficiency [Doerksen et al. 2012; Rattanatranurak et al. 2012; Spafford et al. 2012; Daga et al. 2011].

With regards to computer vision, it is known that the GPU is very effective [Allusse et al. 2008; Babenko and Shah 2008; Fung and Mann 2008; Prisacariu and Reid 2009; Mistry et al. 2011] because of the data-parallel nature of most vision computations. However, as shown, integrated GPUs have different tradeoffs and a GPU-only solution is not efficient here [Lee et al. 2010]. Our code has very high performance comparatively, and we gain a much better or similar fps detection rate compared to recent works on object detection, which use much more capable discrete GPUs [Beleznai et al. 2011; Zhang and Nevatia 2008]. For example, 41fps had been reported using a desktop machine with an Nvidia GTX 260 GPU card [Beleznai et al. 2011], while we achieve $40 \mathrm{fps}$ on a portable device with an integrated on-chip GPU (although comparison is complicated, since the algorithms and machines are different).

Furthermore, trading accuracy for energy or performance has been considered, but in different contexts [Sharrab and Sarhan 2012; Bergman 2010]. For example, Bergman [2010] shows how to limit the processing times for rendering graphics by an OpenGL API library. This method sacrifices frame rate or image quality for less energy consumption. In addition, Sharrab and Sarhan [2012] adapt the video rate for computer vision applications considering both accuracy and power consumption. To gain insight about the accuracy of object detection algorithms, we encourage the reader to go through surveys on similar topics [Zhang and Zhang 2010; Kong et al. 2005; Dollar et al. 2012].

\section{CONCLUSIONS}

Driven by user demand, the computer industry is focused on battery-operated portable devices, which are energy constrained. In addition, better user experience requires natural interfaces using vision and video analytics applications. However, energy-efficient execution of these compute-intensive workloads is challenging.

We showed that heterogeneous on-chip architectures can be very effective using a visual object detection application. We optimized each kernel for the CPU and integrated the GPU of the Ivy Bridge architecture using different techniques. For example, we vectorized the filter kernel using a data layout transformation. 
Furthermore, we showed that a unified programming paradigm such as OpenCL provides a good balance between performance and programmer productivity. This is because the same code runs efficiently on both the CPU and the GPU.

In addition to productivity and performance, energy efficiency is a main concern. By comprehensive evaluation, we showed that it is best to map each kernel where it runs the best. Thus, existing methods, which only use the GPU or try to gain maximum utilization of both the CPU and the GPU naively, are inefficient (even for highly parallel vision workloads). In a nutshell, running each kernel on the best processor type and using software pipelining are both faster and more energy efficient. This is because these heterogeneous on-chip architectures have a fixed chip power budget, which is allocated by a dynamic power management scheme to each processor. If parallelism through software pipelining is not possible, splitting the input among the CPU and GPU might be faster, but specializing each processor for suitable tasks can be more energy efficient.

\section{ACKNOWLEDGMENTS}

This material is based on work supported by the National Science Foundation under Award CNS 1319657 and by the Illinois-Intel Parallelism Center at the University of Illinois at Urbana-Champaign. The center is sponsored by the Intel Corporation. We would like to thank Robert H. Kuhn from Intel and Antonio J. Dios from the University of Malaga (Spain) for their support in the beginning of this project.

\section{REFERENCES}

Allusse, Y., Horain, P., Agarwal, A., and Saipriyadarshan, C. 2008. GpuCV: A GPU-accelerated framework for image processing and computer vision. In Advances in Visual Computing. Lecture Notes in Computer Science Series, vol. 5359. Springer, Berlin, 430-439.

Augonnet, C., Thibault, S., Namyst, R., and Wacrenier, P. 2011. StarPU: A unified platform for task scheduling on heterogeneous multicore architectures. Concurr. Comput. Pract. Exper. 23, 2, 187-198.

Babenko, P. And Shah, M. 2008. MinGPU: A minimum GPU library for computer vision. Journal of Real-Time Image Processing 3, 4, 255-268.

Beleznai, C., Schreiber, D., ANd Rauter, M. 2011. Pedestrian detection using GPU-accelerated multiple cue computation. In Proceedings of the Computer Vision and Pattern Recognition Workshops (CVPRW'11). 58-65.

Bergman, J. 2010. Energy Efficient Graphics: Making the Rendring Process Power Aware. Ph.D. thesis, Uppsala University.

BIK, A. J. C., TIAN, X., AND GIRKAR, M. B. 2006. Multimedia vectorization of floating-point min/max reductions. Concurrency and Computation: Practice and Experience 18, 9, 997-1007.

Bradski, G. 2000. The OpenCV Library. Dr. Dobb's Journal of Software Tools.

BuRGES, C. J. 1998. A tutorial on support vector machines for pattern recognition. Data Mining and Knowledge Discovery 2, 2, 121-167.

Cao, T., Blackburn, S. M., Gao, T., and McKinley, K. S. 2012. The yin and yang of power and performance for asymmetric hardware and managed software. In Proceedings of the International Symposium on Computer Architecture (ISCA'12).

Corbal, J., Espasa, R., AND VAlERo, M. 2001. On the efficiency of reductions in $\mu$-SIMD media extensions. In Proceedings of the International Conference on Parallel Architectures and Compilation Techniques (PACT'01). 83-94.

DAGA, M., AJI, A., AND Feng, W.-C. 2011. On the efficacy of a fused CPU+GPU processor (or APU) for parallel computing. In Proceedings of the Symposium on Application Accelerators in High-Performance Computing (SAAHPC'11). 141-149.

Damaraju, S., George, V., Jahagirdar, S., Khondker, T., Milstrey, R., Sarkar, S., Siers, S., Stolero, I., and SubBiaH, A. 2012. A 22nm ia multi-CPU and GPU system-on-chip. In Proceedings of the IEEE International Solid-State Circuits Conference. 56-57.

David, H., Gorbatov, E., Hanebutte, U. R., Khanna, R., and Le, C. 2010. RAPL: Memory power estimation and capping. In Proceedings of the International Symposium on Low Power Electronics and Design (ISPLED'10). 
Dikmen, M., Hoiem, D., AND Huang, T. S. 2012. A data driven method for feature transformation. In Computer Vision and Pattern Recognition (CVPR'12). IEEE, 3314-3321.

Dikmen, M., Ning, H., Lin, D. J., CaO, L., Le, V., Tsai, S.-F., Lin, K.-H., Li, Z., Yang, J., Huang, T. S., ET AL. 2008. Surveillance event detection. In Proceedings of the TrecVID Video Evaluation Workshop.

Doerksen, M., Thulasiraman, P., and Thulasiram, R. 2012. Optimizing option pricing algorithms and profiling power consumption on VLIW APU architecture. Proc of the 2012 IEEE 10th International Symposium on Parallel and Distributed Processing with Applications (ISPA'12). 71-78.

Dollar, P., Wojek, C., Schiele, B., And Perona, P. 2012. Pedestrian detection: An evaluation of the state of the art. IEEE Transactions on Pattern Analysis and Machine Intelligence 34, 4, 743-761.

Dreslinski, R., Wieckowski, M., Blaauw, D., Sylvester, D., and Mudge, T. 2010. Near-threshold computing: Reclaiming Moore's law through energy efficient integrated circuits. Proceedings of the IEEE 98, 2, 253-266.

Felzenszwalb, P. F., Girshick, R. B., McAllester, D., And Ramanan, D. 2010. Object detection with discriminatively trained part based models. IEEE Transactions on Pattern Analysis and Machine Intelligence 32 , 9, 1627-1645.

Foley, D., Bansal, P., Cherepacha, D., Wasmuth, R., Gunasekar, A., Gutta, S., and Naini, A. 2012. A low-power integrated x86-64 and graphics processor for mobile computing devices. IEEE Journal of Solid-State Circuits 47, 1, 220-231.

FunG, J. AND MANN, S. 2008. Using graphics devices in reverse: Gpu-based image processing and computer vision. In Proceedings of the IEEE International Conference on Multimedia and Expo (ICME'08). 9-12.

González, C. H. and Fraguela, B. B. 2010. A generic algorithm template for divide-and-conquer in multicore systems. In Proceedings of the Conference on High Performance Computing and Communications (HPCC'10). IEEE, 79-88.

Halstead, M. H. 1977. Elements of Software Science (Operating and Programming Systems Series). Elsevier Science Inc.

INTEL. 2011. Avoiding AVX-SSE Transition Penalties. Retrieved November 26, 2013 from http://software. intel.com/en-us/articles/avoiding-avx-sse-transition-penalties.

INTEL. 2013a. Intel SK for OpenCL Applications 2013. Retreived November 26, 2013 from http://software. intel.com/en-us/vcsource/tools/opencl-sdk.

INTEL. 2013b. OpenCL Optimization Guide. Retrieved November 26, 2013 from http://software.intel.com/ sites/products/documentation/ioclsdk/2013/OG/ index.htm.

Jiménez, V. J., Vilanova, L., Gelado, I., Gil, M., Fursin, G., and Navarro, N. 2009. Predictive runtime code scheduling for heterogeneous architectures. In Proceedings of the International Conference on High Performance Embedded Architectures and Compilers (HiPEAC'09).

Jones, M. And Viola, P. 2003. Fast Multi-View Face Detection. Technical Report TR-20003-96 3. Mitsubishi Electronic Research Lab.

Kong, S. G., Heo, J., Aвidi, B. R., PAik, J., And ABidi, M. A. 2005. Recent advances in visual and infrared face recognition a review. Computer Vision and Image Understanding 97, 1, 103-135.

Kumar, R., Tullsen, D. M., Jouppi, N. P., and Ranganathan, P. 2005. Heterogeneous chip multiprocessors. Computer 38, 11.

Lee, V. W., Kim, C., Chhugani, J., Deisher, M., Kim, D., Nguyen, A. D., Satish, N., Smelyanskiy, M., Chennupaty, S., Hammarlund, P., Singhal, R., and Dubey, P. 2010. Debunking the 100x GPU vs. CPU myth: An evaluation of throughput computing on CPU and GPU. In Proceedings of the International Symposium on Computer Architecture (ISCA'10). 451-460.

Lindholm, E., Nickolls, J., Oberman, S., And Montrym, J. March-April 2008. NVIDIA Tesla: A unified graphics and computing architecture. IEEE Micro 28, 2, 39-55.

Liu, C., Li, J., Huang, W., Rubio, J., Speight, E., AND Lin, X. 2012. Power-efficient time-sensitive mapping in heterogeneous systems. In Proceedings of the International Conference on Parallel Architectures and Compilation Techniques (PACT'12).

Luk, C.-K., Hong, S., AND KIM, H. 2009. Qilin: exploiting parallelism on heterogeneous multiprocessors with adaptive mapping. In Proceedings of the IEEE/ACM International Symposium on Microarchitecture (MICRO'09).

MA, K., Li, X., Chen, W., Zhang, C., AND WanG, X. 2012. GreenGPU: A holistic approach to energy efficiency in GPU-CPU heterogeneous architectures. In Proceedings of the International Conference on Parallel Processing (ICPP'12).

MA, T. AND LATECKI, L. J. 2011. From partial shape matching through local deformation to robust global shape similarity for object detection. In Proceedings of the Conference on Computer Vision and Pattern Recognition (CVPR'11). IEEE, 1441-1448. 
MAJI, S. AND MALIK, J. 2009. Object detection using a max-margin hough transform. In Computer Vision and Pattern Recognition. IEEE, 1038-1045.

Maleki, S., Gao, Y., Garzarán, M. J., Wong, T., and Padua, D. A. 2011. An evaluation of vectorizing compilers. In Proceedings of the International Conference on Parallel Architectures and Compilation Techniques (PACT'11). 372-382.

Mistry, P., GregG, C., Rubin, N., Kaeli, D., and Hazelwood, K. 2011. Analyzing program flow within a manykernel openCL application. In Proceedings of the 4th Workshop on General Purpose Processing on Graphics Processing Units (GPGPU'11).

Mudge, T. 2001. Power: A first-class architectural design constraint. Computer 34, 4, 52-58.

NVIDIA. 2011. Bringing High-End Graphics to Handheld Devices. Retrieved November 26, 2013 from http://www.nvidia.com/content/PDF/tegra_white_papers/Bringing_High-End_Graphics_to_Handheld_ Devices.pdf.

Planas, J., Badia, R. M., Ayguade, E., and Labarta, J. 2013. Self-adaptive OMPSS tasks in heterogeneous environments. In Proceedings of the IEEE International Parallel \& Distributed Processing Symposium (IPDPS'13).

Prisacariu, V. AND ReID, I. 2009. FastHog-A Real-Time GPU Implementation of Hog. Technical Report 2310 , 09. University of Oxford.

Rattanatranurak, A., Kittitornkun, S., and Tongsima, S. 2012. Optimizing and multithreading snphap on a multi-core apu with opencl. In Proceedings of International Joint Conference on Computer Science and Software Engineering (JCSSE'12). 174-179.

REINDERS, J. 2007. Intel Threading Building Blocks, 1st ed.

Ren, G., Wu, P., ANd Padua, D. 2006. Optimizing data permutations for SIMD devices. In Proceedings of the ACM SIGPLAN Conference on Programming Language Design and Implementation (PLDI'06). 118-131.

Rotem, E., Naveh, A., Rajwan, D., Ananthakrishnan, A., and Weissmann, E. March-April 2012. Powermanagement architecture of the Intel microarchitecture code-named sandy bridge. IEEE Micro 32, $2,20-27$.

SharRab, Y. AND SARHAN, N. 2012. Accuracy and power consumption tradeoffs in video rate adaptation for computer vision applications. In Proceedings of the International Conference on Multimedia and Expo (ICME'12). 410-415.

Spafford, K., Meredith, J., Lee, S., Li, D., Roth, P., and Vetter, J. 2012. The tradeoffs of fused memory hierarchies in heterogeneous computing architectures. In Proceedings of the Conference on Computing Frontiers (CF'12). 103-112.

Talla, D., John, L., And Burger, D. Aug. 2003. Bottlenecks in multimedia processing with SIMD style extensions and architectural enhancements. IEEE Transaction on Computers 52, 8, 1015-1031.

Thoman, P., Kofler, K., Studt, H., Thomson, J., and Fahringer, T. 2011. Automatic openCL device characterization: Guiding optimized kernel design. In Euro-Par 2011. 438-452.

WEYUKER, E. J. 1988. Evaluating software complexity measures. IEEE Transactions on Software Engineering $14,9,1357-1365$.

YANG, M., Ji, S., Xu, W., Wang, J., Lv, F., Yu, K., Gong, Y., Dikmen, M., Lin, D. J., AND Huang, T. S. 2009. Detecting human actions in surveillance videos. In Proceedings of the TrecVID Video Evaluation Workshop.

Zhang, C. And Zhang, Z. 2010. A Survey of Recent Advances in Face Detection. Technical Report MSR-TR2010-66.

Zhang, L. And Nevatia, R. 2008. Efficient scan-window based object detection using gpgpu. In Proceedings of the Computer Vision and Pattern Recognition Workshops (CVPRW'08). 1-7.

Zhang, Y., Hu, Y., Li, B., AND Peng, L. 2011. Performance and power analysis of ATI GPU: A statistical approach. In Proceedings of the IEEE International Conference on Networking, Architecture and Storage (NAS'11). 149-158.

Received June 2013; revised September 2013; accepted November 2013 